\title{
Highly Purified Cannabidiol for Epilepsy Treatment: A Systematic Review of Epileptic Conditions Beyond Dravet Syndrome and Lennox-Gastaut Syndrome
}

\author{
Simona Lattanzi ${ }^{1}$ D Eugen Trinka ${ }^{2,3,4} \cdot$ Pasquale Striano $^{5} \cdot$ Chiara Rocchi $^{1} \cdot$ Sergio Salvemini $^{1} \cdot$ Mauro Silvestrini $^{1}$. \\ Francesco Brigo ${ }^{6,7}$
}

Accepted: 10 March 2021 / Published online: 22 March 2021

(c) The Author(s) 2021

\begin{abstract}
Background Cannabidiol (CBD), which is one major constituent of the Cannabis sativa plant, has anti-seizure properties and does not produce euphoric or intrusive side effects. A plant-derived, highly purified CBD formulation with a known and constant composition has been approved by the US Food and Drug Administration for the treatment of seizures associated with Dravet syndrome, Lennox-Gastaut syndrome, and tuberous sclerosis complex. In the European Union, the drug has been authorized by the European Medicines Agency for the treatment of seizures associated with Dravet syndrome and Lennox-Gastaut syndrome, in conjunction with clobazam, and is under regulatory review for the treatment of seizures in patients with tuberous sclerosis complex.

Objectives This systematic review aimed to summarize the currently available body of knowledge about the use of this US Food and Drug Administration/European Medicines Agency-approved oral formulation of pharmaceutical-grade CBD in patients with epileptic conditions, especially developmental and epileptic encephalopathies other than Dravet syndrome and Lennox-Gastaut syndrome.

Methods The relevant studies were identified through MEDLINE and the US National Institutes of Health Clinical Trials Registry in October 2020. There were no date limitations or language restrictions. The following types of studies were included: clinical trials, cohorts, case-control, cross-sectional, clinical series, and case reports. Participants had to meet the following criteria: any sex, any ethnicity, any age, diagnosis of epilepsy, receiving plant-derived, highly purified ( $>98 \%$ w/w) CBD in a sesame oil-based oral solution for the treatment of seizures. Data extracted from selected records included efficacy, tolerability, and safety outcomes.

Results Five hundred and seventy records were identified by database and trial register searching. Fifty-seven studies were retrieved for detailed assessment, of which 42 were eventually included for the review. The participants of the studies included patients of both pediatric and adult age. Across the trials, purified CBD was administered at dosages up to 50 mg/ $\mathrm{kg}$ /day. In a randomized double-blind controlled trial in patients with tuberous sclerosis complex, CBD was associated with a significantly greater percent reduction in seizure frequency than placebo over the treatment period. Open-label studies suggested the effectiveness of CBD in the treatment of children and adults presenting with other epilepsy syndromes than those addressed by regulatory trials, including CDKL5 deficiency disorder and Aicardi, Dup15q, and Doose syndromes, SYNGAP1 encephalopathy, and epilepsy with myoclonic absences. The most common adverse events observed during treatment with CBD included somnolence, decreased appetite, diarrhea, and increased serum aminotransferases.

Conclusions The currently available data suggest that response to treatment with a highly purified, plant-derived CBD oilbased solution can be seen in patients across a broad range of epilepsy disorders and etiologies. The existing evidence can provide preliminary support for additional research.
\end{abstract}

Extended author information available on the last page of the article 


\section{Key Points}

Pharmaceutical-grade cannabidiol (CBD) represents the first in a new class of antiseizure medications

In randomized controlled trials, CBD reduced seizure frequency in patients with Dravet syndrome, LennoxGastaut syndrome, and tuberous sclerosis complex

Open-label studies suggest the effectiveness of CBD treatment in patients with other epileptic conditions than those addressed by regulatory trials

The most common adverse events associated with CBD include somnolence, decreased appetite, diarrhea, and increased serum aminotransferases with DS and LGS, in conjunction with clobazam (CLB), in patients aged $\geq 2$ years and is under regulatory review for the treatment of seizures in patients with TSC. This is the first in a new class of antiseizure medications and the only pharmaceutical formulation derived from the cannabis plant that has undergone review through the approval processes and received marketing authorization for these difficult-totreat epileptic syndromes.

Clinical evidence about CBD treatment in patients with DS and LGS has been already reviewed [10-12]. Here, we summarize the currently available body of knowledge about the use of this Food and Drug Administration/European Medicines Agency-approved oral formulation of pharmaceutical-grade CBD in patients with other epileptic conditions and suggest implications for clinical practice and future research.

\section{Methods}

The results of this systematic review were reported according to the recommendations of the Preferred Reporting Items for Systematic Reviews and Meta-Analyses (PRISMA) statement.

The relevant studies were identified through MEDLINE (accessed by PubMed as of October 2020, week 4) and the US National Institutes of Health Clinical Trials Registry (http://www.clinicaltrials.gov). Search strategies are outlined in the Electronic Supplementary Material (ESM). There were no date limitations or language restrictions. The protocol was not registered previously.

The following types of studies were included: clinical trials, cohorts, case-control, cross-sectional, clinical series, and case reports. Self-reported surveys, reviews, meta-analyses, editorials, commentaries, and expert opinions were excluded. Participants had to meet the following criteria: any sex, any ethnicity, any age, diagnosis of epilepsy, receiving plant-derived, highly purified ( $>98 \% \mathrm{w} / \mathrm{w}$ ) CBD in a sesame oil-based oral solution (Epidiolex/Epidyolex ${ }^{\circledR}$ ) for the treatment of seizures. We excluded studies that recruited only patients with LGS or DS, non-epilepsy disorders (e.g., pain, sleep), or patients with non-epileptic seizure types (e.g., non-epileptic myoclonus such as Lance-Adams syndrome). The drug manufacturer was contacted for information about any unpublished or ongoing studies. Reference lists of the selected articles were reviewed to identify additional reports of relevant studies.

Two review authors independently assessed studies for inclusion and extracted the following information from included studies: main study author and age of publication, number and demographics of participants, seizure outcomes (e.g., seizure frequency, $50 \%$ responder rate, seizure-free 
rate, seizure severity), safety outcomes (e.g., adverse event rate, rates of individual adverse events), and other effectiveness and clinical outcomes (e.g., retention rate, cognitive function, behavior, mood, brain imaging). Any disagreement was resolved by discussion with a third review author.

\section{Results}

Five hundred and seventy records were identified by database and trial register searching. Fifty-seven studies were retrieved for detailed assessment, of which 42 were eventually included for the review (Fig. 1). The included studies were randomized placebo-controlled trials [13, 43, 47], open-label interventional studies and their subgroup analyses [14, 15, 17, 18, 20-24, 26-30, 32, 34-38, 41, 42, 48-52], retrospective chart reviews [39, 44, 46], clinical series [19, $40,53]$, and case reports [16, 25, 31, 33, 45, 54]. The participants of the studies included patients of pediatric age [14-17, 19, 28, 30, 31, 40, 41, 45, 46, 50, 52, 53], adult age $[25,32,33,36,47,48,51,54]$, and both pediatric and adult age [13, 18, 20-24, 26, 27, 29, 34, 35, 37-39, 42-44, 49]. Details of the characteristics of participants and outcomes of the included studies are provided in the ESM.

Across the trials, purified CBD was administered as adjunctive treatment at dosages up to $50 \mathrm{mg} / \mathrm{kg} /$ day. Most studies aimed to assess the efficacy and safety of CBD treatment, and study endpoints included seizure frequency reduction, seizure response rate, seizure freedom, change in seizure severity, treatment discontinuation, and occurrence of adverse events. Eight studies were primarily aimed to describe pharmacokinetic analyses or drug-drug interactions between CBD and antiseizure or non-antiseizure

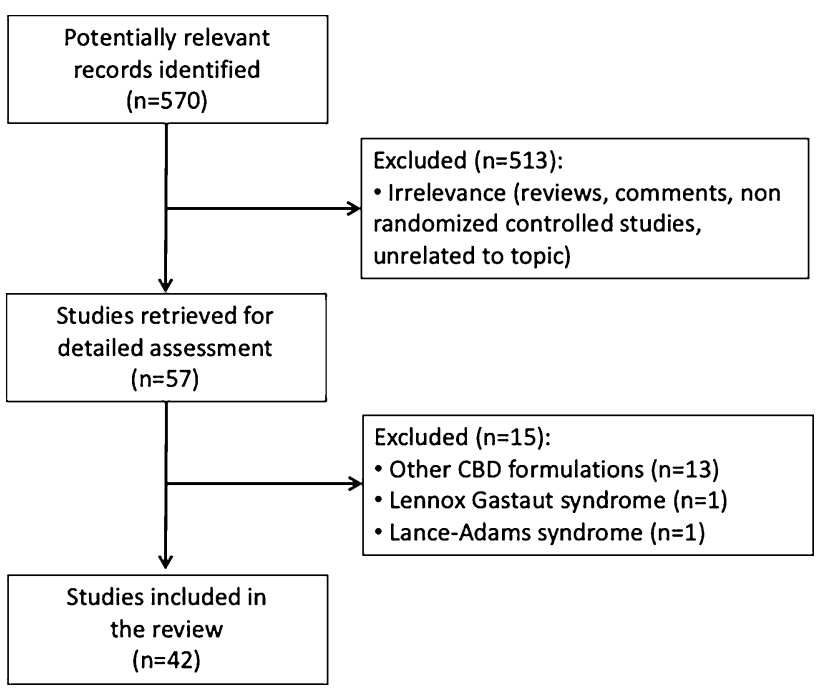

Fig. 1 Flow diagram of the study selection process. $C B D$ cannabidiol medications [17, 23, 25, 33, 39, 43, 47, 54]. Seven studies included cognitive and/or quality-of-life measures [20-22, $32,36,50,51]$, and three mainly focused on functional brain imaging assessment $[35,49,51]$.

18 studies provided outcome data according to individual epileptic conditions or etiologies, including TSC, CDKL5 deficiency, Aicardi syndrome, Doose syndrome, Dup15q syndrome, epilepsy with myoclonic absences, Sturge-Weber syndrome, SYNGAP1 developmental and epileptic encephalopathy, epilepsy of infancy with migrating focal seizures, SCN8A-related epilepsy with encephalopathy, epileptic spasms, focal cortical dysplasia, cerebral dysgenesis, and lissencephaly; efficacy and tolerability data were also available for patients presenting with febrile infection-related epilepsy syndrome, and status epilepticus (Table 1). Twenty-four studies included patients with uncontrolled or treatmentresistant epilepsy of different etiologies, which were listed or not in the original papers, and outcomes were described for the whole cohorts without details for individual conditions (Table 2).

\section{Discussion}

The existing data suggest that response to treatment with a highly purified, plant-derived CBD oil-based solution can be seen in patients across a broad range of epilepsy disorders and etiologies.

The highest-quality evidence is currently available for TSC. In one randomized, double-blind, controlled trial, $\mathrm{CBD}$ at both the dosages of 25 and $50 \mathrm{mg} / \mathrm{kg} /$ day produced a significantly greater percent reduction in TSC-associated focal and generalized seizure frequency and total seizure frequency, including focal sensory and epileptic spasms than placebo over the treatment period. The $50 \%$ responder rate among patients taking CBD was around $40 \%$ and substantially overlapped the rate observed in patients with TSC who were recruited in a prior expanded access study of CBD up-titrated to a maximum dose of $50 \mathrm{mg} / \mathrm{kg} /$ day within the frame of treatment-resistant epilepsies [20]. Further, the administration of purified CBD as adjunctive therapy at a dosage range of $10-50 \mathrm{mg} / \mathrm{kg} /$ day resulted in electrographic and clinical response in patients with TSC with refractory epileptic spasms enrolled in an open-label study: a reduction in epileptic spasm frequency was observed after 2 weeks of treatment, resolution of hypsarrhythmia correlated with the reduction in epileptic spasms, and all patients were free from epileptic spasms at 12 months [41]. Subjective improvements in cognitive and behavioral domains were also seen alongside improvement of background activity [41].

Open-label drug trials provide class III evidence for the efficacy of CBD administration in patients with CDKL5 deficiency disorder and Aicardi, Doose, and Dup15q 
Table 1 Main clinical outcomes according to epilepsy condition or etiology

\begin{tabular}{ll}
\hline Epilepsy condition/etiology & Population \\
\hline Tuberous sclerosis complex & $N=224(\mathrm{CBD} 25: n=75, \mathrm{CBD} 50 n=73$, \\
& placebo $n=76)[13] ; N=2 ;[17] N=$ \\
& $18[20]$ \\
& $N=25[39] ; N=3[41]$
\end{tabular}

Main findings

Randomized, double-blind, placebo-controlled trial Reduction in TSC-associated seizure frequency: 48.6\% (CBD25; $p<0.001), 47.5 \%$ (CBD50; $p=$ $0.002), 26.5 \%$ (placebo); responder rate TSCassociated seizure frequency: $36.0 \%$ (CBD25; $p=0.069), 39.7 \%$ (CBD50; $p=0.025), 22.4 \%$ (placebo); reduction in total seizure frequency: $48 \%$ $(\mathrm{CBD} 25 ; p=0.001), 48 \%(\mathrm{CBD} 50 ; p=0.002)$, $27 \%$ (placebo) during 16-week treatment period. Improvement in overall condition on S/CGIC: $69 \%$ $(\mathrm{CBD} 25 ; p=0.007), 62 \%(\mathrm{CBD} 50 ; p=0.058)$, $40 \%$ (placebo)

Treatment withdrawal: 10.3\%. AEs: $88.0 \%$ (CBD25), 97.3\% (CBD50), $89.5 \%$ (placebo); diarrhea: 30.7\% (CBD25), $54.8 \%$ (CBD50), 25.0\% (placebo); decreased appetite: $20.0 \%$ (CBD25), $23.3 \%$ (CBD50), 11.8\% (placebo); somnolence: $13.3 \%$ (CBD25), 26.0\% (CBD50), 9.2\% (placebo); vomiting: $16.0 \%$ (CBD25), $17.8 \%$ (CBD50), 9.2\% (placebo); pyrexia: $18.7 \%$ (CBD25), 16.4\% (CBD50), $7.9 \%$ (placebo); increased transaminases: $12.0 \%$ (CBD25), 24.7\% (CBD50), 0\% (placebo). SAEs: 21.3\% (CBD25), $13.7 \%$ (CBD50), 2.6\% (placebo) [13]

Open-label studies

Seizure frequency reduction: 58-93\% (week 8) [17]

Reduction in epileptic spasm frequency: 15.1-98.8\% (week 2), 3.7-94.2\% (M1), 58.3-100\% (M2), 49.1-100\% (M3), 80.2-100\% (M6), 85.8-100\% (M9), 100\% (M12) [41]

Seizure frequency reduction $>50 \%: 50 \%$ (M2), 50\% (M3), 38.9\% (M6), 50\% (M9), and 50\% (M12) for all seizure types; $100 \%$ (M2), $75 \%$ (M3), $100 \%$ (M6), $100 \%$ (M9), and 100\% (M12) for spasms; $75 \%$ (M2), $75 \%$ (M3), 75\% (M6), 75\% (M9), and $50 \%$ (M12) for atonic seizures; 50\% (M2), 66.7\% (M3), 50\% (M6), 60\% (M9), and 100\% (M12) for tonic clonic seizures; $38.5 \%$ (M2), 53.8\% (M3), $30.8 \%$ (M6), 53.8\% (M9), and 50\% (M12) for focal seizures with impairment of consciousness or awareness; 25\% (M2), 50\% (M3), 50\% (M6), 66.7\% (M9), and 50\% (M12) for focal seizures evolving to bilateral generalized convulsive seizures; $57.1 \%$ (M2), 42.9\% (M3), 57.1\% (M6), 66.7\% (M9), and $50 \%$ (M12) for tonic seizures [20]

Treatment withdrawal: $16.7 \%$ [20]

Resolution of hypsarrhythmia: 2/2 [41]

Cognitive gains 12/14 (85.7\%), behavioral improvement 6/9 (66.7\%) [20]

AEs: $54.2 \%$. Drowsiness $22.9 \%$, diarrhea $14.6 \%$, ataxia $12.5 \%$, agitation $10.4 \%$, irritability $6.3 \%$, lethargy $6.3 \%$, appetite loss $4.2 \%$, poor sleep $4.2 \%$, confusion, vomiting, abdominal pain, mouth sores, increased acne, ankle swelling, sinusitis, mild elevation of transaminases, increased phenytoin level, increased self-stimulation, behavioral difficulties (all 2.1\%) [17, 20, 39, 41]

CBD daily dose: $15-50 \mathrm{mg} / \mathrm{kg}$ [20]; 5-20 mg/kg [39] 
Table 1 (continued)

\section{Epilepsy condition/etiology}

Aicardi syndrome

CDKL5 deficiency

Doose syndrome

Dup15q syndrome

Epilepsy with myoclonic absences

Sturge-Weber syndrome
$N=1[17] ; N=20[27] ; N=5[30]$

$N=2[17] ; N=8[27]$

$N=1[17] ; N=8$ [27]

$N=5[30]$

$N=5[21]$
Main findings

Median decrease in convulsive seizure frequency: $58.3 \%$ (week 12), 59.2\% (week 48). Responder rate (convulsive seizures): $71 \%$ (week 12), 71\% (week 48). Mean CBD daily dose: $8.1 \pm 2.3$ (week 2 ), $26.7 \pm 12.7$ (week 12), $32.0 \pm 12.3$ (week 48) mg/ $\mathrm{kg}$

Seizure frequency reduction: 100\% (week 8) [17] Median decrease in convulsive seizure frequency: $40.8 \%$ (week 12), 59.7\% (week 48). Responder rate (convulsive seizures): $41 \%$ (week 12), 53\% (week 48) [27]

Responder rate $1 / 5$, increased seizure frequency $2 / 5$ (any seizure types; 8-36 months) [30]

Increased seizure frequency: (any seizure types): $2 / 5$ [30]

AEs: 4/5. Drowsiness, weight loss, sleepiness, diarrhea, loose stools, agitation. Treatment withdrawal: $4 / 5$ (at 5-23 months) [30]

Mean CBD daily dose: $8.3 \pm 2.6$ (week 2$), 18.2 \pm 7.0$ (week 12), $26.2 \pm 10.1$ (week 48) mg/kg [27]

Seizure frequency reduction: $54-100 \%$ (week 8). AEs: 50\% (drowsiness) [17]

Median decrease in convulsive seizure frequency: $58.6 \%$ (week 12), 28.8\% (week 48). Convulsive seizures responder rate: $43 \%$ (week 12), $57 \%$ (week 48) [27]. Mean CBD daily dose: $8.8 \pm 2.5$ (week 2), $22.0 \pm 5.3$ (week 12), $27.5 \pm 15.5$ (week 48) $\mathrm{mg} / \mathrm{kg}$ [27]

Seizure frequency reduction: $26 \%$ (week 8) [17] Median decrease in convulsive seizure frequency: $25.0 \%$ (week 12), 38.4\% (week 48). Convulsive seizures responder rate: $38 \%$ (week 12), 38\% (week 48) [27]

AEs: 1/1; ataxia, tremor, loss of appetite [17]. Mean CBD daily dose: $8.7 \pm 1.9$ (week 2), $18.4 \pm 7.4$ (week 12), $29.2 \pm 9.1$ (week 48) mg/kg [27]

Responder rate $2 / 5$, seizure freedom $2 / 5$, increased seizure frequency: 2/5 (any seizure types; 5-53 months)

Treatment withdrawal: 3/5 (at 5-6 months) AEs: 5/5; decreased appetite/food aversion, weight loss, elevated transaminases, loose stools, lethargy

Seizure frequency reduction: 10-90\% (week 14), 12-100\% (at most recent visit - week 6-60).

Treatment withdrawal for lack of efficacy: $2 / 5$ (at week 9 and 38)

AEs: $5 / 5$; temporary increased seizures (3/5), behavioral issues $(2 / 5)$, increased transaminases $(1 / 5)$, tiredness $(1 / 5)$

All patients reported improvements in quality of life; subjective improvements in motor, speech, and cognitive abilities, level of alertness, vocalization or communication, mood and behavior also reported. CBD daily dose: $5-25 \mathrm{mg} / \mathrm{kg}$ 
Table 1 (continued)

\begin{tabular}{ll}
\hline Epilepsy condition/etiology & Population \\
\hline $\begin{array}{l}\text { SYNGAP1 developmental and epileptic } \\
\text { encephalopathy }\end{array}$ & $N=3[53]$ \\
& \\
Epilepsy of infancy with migrating focal & $N=1[16] ; N=1[30] ; N=2[40]$ \\
seizures &
\end{tabular}
seizures

SCN8A epileptic encephalopathy

Infantile/epileptic spasms
$N=1[30]$

$N=9[14] ; N=9[15] ; N=6[41]$
Focal cortical dysplasia
$N=2[49]$
Main findings

Seizure frequency reduction: 0-85\% (M2), 80-95\%

(M9). Responder rate: 2/3 (M2), 3/3 (M9)

AEs: $1 / 3$ (sleep disorder). Slight increase in transaminases $(1 / 3)$

EEG improvement in background activity and interictal anomalies

Caregivers evaluated as much improved the status of their children. Maximum CBD daily dose: 10-23 $\mathrm{mg} / \mathrm{kg}$

Reduction in seizure frequency $>90$ at M6, with seizure-free periods; improvement in alertness [16]

CBD discontinued after 6 months for inefficacy [30]

Overall seizure frequency change $-12 \%$ to $+20 \%$ during 24-week treatment period; increase in motor arrest seizures with clinically meaningful reduction in seizure intensity, reduction in generalized clonic seizures, reduction/increase in asymmetric tonic seizures [40]

AEs: $75 \%$. Somnolence 2/4, intermittent vomiting 1/4 [16, 30, 41]. Maximum CBD daily dose: $25 \mathrm{mg} / \mathrm{kg}$ $[16,40]$

CBD dose reduced for side effects and discontinued after 25 months for inefficacy

AEs: drowsiness, somnolence

Day 15: freedom of clinical spasms 0/9; resolution of hypsarrhythmia 0/9; improvement at CGIC 7/9; improvement at PGIC 6/9; AEs 5/9 (55.6\%); diarrhea $2 / 9(22.2 \%)$, upper respiratory tract infection 2/9 (22.2\%), somnolence 1/9 (11.1\%); serious AEs $1 / 9(11.1 \%)$ [14]

Freedom of clinical spasms: 1/8 (day 29), 2/6 (day 43), 1/4 (day 127), 1/3 (day 211), 1/2 (day 295), 3/7 (day 379). Resolution of hypsarrhythmia: 1/8 (day 29), 0/6 (day 43), 1/4 (day 127), 0/3 (day 211), 1/2 (day 295), 3/7 (day 379). Freedom of clinical spasms and resolution of hypsarrhythmia: $1 / 8$ (day 29), 0/6 (day 43), 0/4 (day 127), 0/3 (day 211), 1/2 (day 295), 3/7 (day 379). Improvement at CGIC: 7/9 (day 29), 6/9 (day 43), 4/9 (day 127), 3/9 (day 211), 3/9 (day 295), 6/9 (day 379); improvement at PGIC: 4/9 (day 29), 5/9 (day 43), 4/9 (day 127), 1/9 (day 211), 1/9 (day 295), 4/8 (day 379). AEs (day 417): 7/9 (77.8\%); SAEs (day 417): 2/9 (22.2\%) [15]

Change in epileptic spasm frequency: $-100 \%$ to $10.6 \%$ (week 2), $-96.0 \%$ to $10.6 \% \%$ (M1), -100 to $9.6 \%$ (M2), -100 to $12.3 \%$ (M3), -100 to $-29.8 \%$ (M6), -100 to $-76.2 \%$ (M9), -100 to $-42.4 \%$ (M12). Resolution of hypsarrhythmia: 3/6. Improvement in cognitive and developmental motor skills: 2/6 [41]. Maximum CBD daily dose: $40 \mathrm{mg} /$ $\mathrm{kg}[14,15]$

Seizure frequency reduction from 70 to $100 \%$, seizure freedom in $1 / 2$ after receiving a stable dosage of CBD for at least 2 weeks. CBD daily dose: 15-20 $\mathrm{mg} / \mathrm{kg}$ 
Table 1 (continued)

\begin{tabular}{ll}
\hline Epilepsy condition/etiology & Population \\
\hline Cerebral dysgenesis & $N=3[17] ; N=1[41]^{\mathrm{a}}$ \\
& \\
\hline Lissencephaly & $N=1[17]$ \\
Tumor-related epilepsy & $N=3[24] ; N=2[49]$
\end{tabular}

Febrile infection-related epilepsy syndrome $\quad N=7$ [19]

Super refractory status epilepticus $\quad N=1$ [31]

$N=1[45]$

Rett syndrome

Refractory generalized epilepsy
$N=1[30]$

$N=1[30] ; N=2[49]$
Main findings

Change in seizure frequency: $-74 \%$ to $99 \%$ (week 8); increase in seizure frequency: $2 / 3$ [17]

Reduction in epileptic spasm frequency: $100 \%$ (week 2), $92.5 \%$ (M1), $82.5 \%$ (M2), $60 \%$ (M3), $67.5 \%$ (M6), 85\% (M9), 85\% (M12) and resolution of hypsarrhythmia [41]

AEs: 4/4. Drowsiness (3/4), ataxia and urinary retention (1/4) [17]

Seizure frequency reduction: $94 \%$ (week 8). AEs: none

Seizure frequency reduction on-study 58-94\% (month 2-11) in 2/3

Seizure frequency reduction from 57 to $86 \%$ after receiving a stable dosage of $\mathrm{CBD}$ for at least 2 weeks [49]

Seizure frequency increase in $1 / 5[24,49]$

Improvement in seizure severity as assessed by CSSS (3/3) and total Mood Disturbance score (2/3) [24]. CBD daily dose: $20-50 \mathrm{mg} / \mathrm{kg}$ [24]; $25 \mathrm{mg} / \mathrm{kg}$ [49]

Resolution of status epilepticus in 1 out of 2 patients treated in the acute phase

Mean seizure frequency reduction in 5 patients treated in the chronic phase: $90.9 \%$ (week 4) and $65.3 \%$ (week 48) for all seizure types, $99.6 \%$ (week 4) and $62.3 \%$ (week 48) for focal motor seizures, $75 \%$ (week 4) and $73 \%$ (week 48) for generalized tonic-clonic seizures, $99.6 \%$ (week 4) and $62.4 \%$ (week 48 ) for focal seizures with impaired consciousness

AEs: dizziness (2/7), decreased appetite and weight loss (1/7), nausea/vomiting (1/7). CBD daily dose: $15-25 \mathrm{mg} / \mathrm{kg}$

Clinical seizure freedom achieved on day 12, clinical and subclinical seizure freedom demonstrated on day 64; sequential discontinuation of phenobarbital, midazolam, perampanel, and dose-reduction of lacosamide [31]

Reduction of frequency in clinical seizures (from 10 to $0-3$ episodes per hour) and midazolam drip successfully weaned off [45]

AEs: fatigue, weight gain [31]. Maximum CBD daily dose: $20 \mathrm{mg} / \mathrm{kg}$ [31]; $25 \mathrm{mg} / \mathrm{kg}$ [45]

CBD dose reduced for side effects and discontinued after 6 months for inefficacy. AEs: agitation, insomnia, leg cramping

Reduction $>50 \%$ in seizures frequency: $1 / 1$ (generalized tonic-clonic), 1/1 (tonic seizures) after 43 months of CBD treatment [30] AEs: decreased appetite, weight loss, diarrhea, elevated transaminases [30]

Change in seizure frequency: -20.8 to $42.8 \%$ after receiving a stable dosage of CBD for at least 2 weeks [49]. CBD daily dose: $20 \mathrm{mg} / \mathrm{kg}$ [49] 
Table 1 (continued)

\begin{tabular}{|c|c|c|}
\hline Epilepsy condition/etiology & Population & Main findings \\
\hline Refractory focal epilepsy & $N=2[30] ; N=13[49]$ & $\begin{array}{l}\text { Focal epilepsy (vasculitis). Seizure frequency reduc- } \\
\text { tion }>50 \%: 1 / 1 \text { at } 40 \text { months; AE: none [30] } \\
\text { Focal epilepsy (unknown etiology). Seizure fre- } \\
\text { quency reduction }>50 \%: 0 / 1 \text {; treatment withdrawal } \\
1 / 1 \text { (at } 5 \text { months); AE: diarrhea [30] } \\
\text { Focal epilepsy (encephalitis; } n=2 \text { ): change in } \\
\text { seizure frequency }-53.9 \text { to }-42.9 \% \text { after receiving } \\
\text { a stable dosage of CBD for at least } 2 \text { weeks [49]. } \\
\text { CBD daily dose: } 25 \mathrm{mg} / \mathrm{kg} \text { [49] } \\
\text { Multifocal epilepsy (unknown etiology; } n=1 \text { ): } \\
\text { change in seizure frequency }-100 \% \text { after receiving } \\
\text { a stable dosage of CBD for at least } 2 \text { weeks [49]. } \\
\text { CBD daily dose: } 25 \mathrm{mg} / \mathrm{kg} \text { [ } 49 \text { ] } \\
\text { Temporal lobe epilepsy (unknown etiology; } n= \\
\text { 7): change in seizure frequency }-100 \% \text { to } 50 \% \text {, } \\
\text { responder rate } 5 / 7, \text { seizure freedom } 2 / 7 \text { after receiv- } \\
\text { ing a stable dosage of CBD for at least } 2 \text { weeks } \\
\text { [49]. CBD daily dose: } 15-25 \mathrm{mg} / \mathrm{kg}[49] \\
\text { Frontal lobe epilepsy (unknown etiology; } n=4 \text { ): } \\
\text { change in seizure frequency }-100 \% \text { to } 14.3 \% \text {, } \\
\text { responder rate } 3 / 4, \text { seizure freedom } 1 / 4 \text { after receiv- } \\
\text { ing a stable dosage of CBD for at least } 2 \text { weeks } \\
\text { [49]. CBD daily dose: } 15-25 \mathrm{mg} / \mathrm{kg}[49]\end{array}$ \\
\hline
\end{tabular}

$A E$ adverse event, $C B D$ cannabidiol, $C B D 25$ cannabidiol $25 \mathrm{mg} / \mathrm{kg} /$ day, $C B D 50$ cannabidiol $50 \mathrm{mg} / \mathrm{kg} /$ day, CSSS Chalfont Seizure Severity Scale, $E E G$ electroencephalogram, $M 1$ month 1, M2 month 2, M3 month 3, M6 month 6, M9 month 9, M12 month 12, SAE serious adverse event, $S / C G I C$ subject/caregiver global impression of change

${ }^{a}$ Patient with West syndrome history

syndromes, which are common causes of epileptic encephalopathies. Add-on treatment with pure CBD was associated with a significant reduction in baseline monthly seizure frequency at week 12 , and no difference in seizure percent change was observed between week 12 and week 48, suggesting sustained efficacy. Long-term response to treatment was further reported over 2-4 years in patients with medically refractory epilepsy, including CDKL5 epileptic encephalopathy and epilepsy with myoclonic absences [30]. Remarkably, patients enrolled in these studies were among the most treatment-resistant patients at each center and had syndromes characterized by a poor outcome, with a high incidence of status epilepticus, use of rescue medication, and sudden unexpected death in epilepsy. They had high baseline seizure frequency and a number of concomitant antiseizure medications, and many had never obtained seizure control despite pharmacological and non-pharmacological interventions, including vagus nervus stimulation, dietary changes, and surgical treatments. In this scenario, the rates of seizure response and even seizure freedom for a few cases are suggestive of clinically relevant CBD efficacy.

A significant decrease in seizure frequency from 80 to 95\% has been observed with CBD add-on therapy in patients with SYNGAP1 developmental and epileptic encephalopathy, accompanied by an improvement of background electroencephalogram activity and interictal anomalies. The reduction in drop attacks and myoclonic seizures, which are associated with a high risk of seizure-related injuries [55], could determine a favorable effect on the general condition of the patients. Interestingly, syngap1 heterozygous mutations induce an increase in the transient receptor potential cation channel subfamily $\mathrm{V}$ member 1 protein, which can be one of the mechanisms underlying the excitatory/inhibitory imbalance in drug-resistant epilepsy related to SYNGAP1 mutations [56, 57], and CBD has been shown to induce a rapid activation and desensitization of the transient receptor potential cation channel subfamily $\mathrm{V}$ member 1 [58].

One randomized, double-blind, placebo-controlled trial to investigate the efficacy and safety of CBD titrated to a target dose of $40 \mathrm{mg} / \mathrm{kg} /$ day in patients with infantile spasms aged 1-24 months had its primary endpoint measure based on an electroencephalogram and was stopped as it met the prespecified "No-Go Criteria" in its pilot phase after the recruitment of nine participants; improvement in caregiver and physician global impression of change was observed in most cases [14]. Clinical improvement following add-on CBD treatment was, instead, clearly observed in children aged between 3 and 16 years who presented with refractory epileptic spasms and participated in one open-label study: around $70 \%$ of patients were responders at 2 weeks, one third were free from epileptic spasms after 2 months, and $60 \%$ had a resolution in their hypsarrhythmia pattern [41]. 


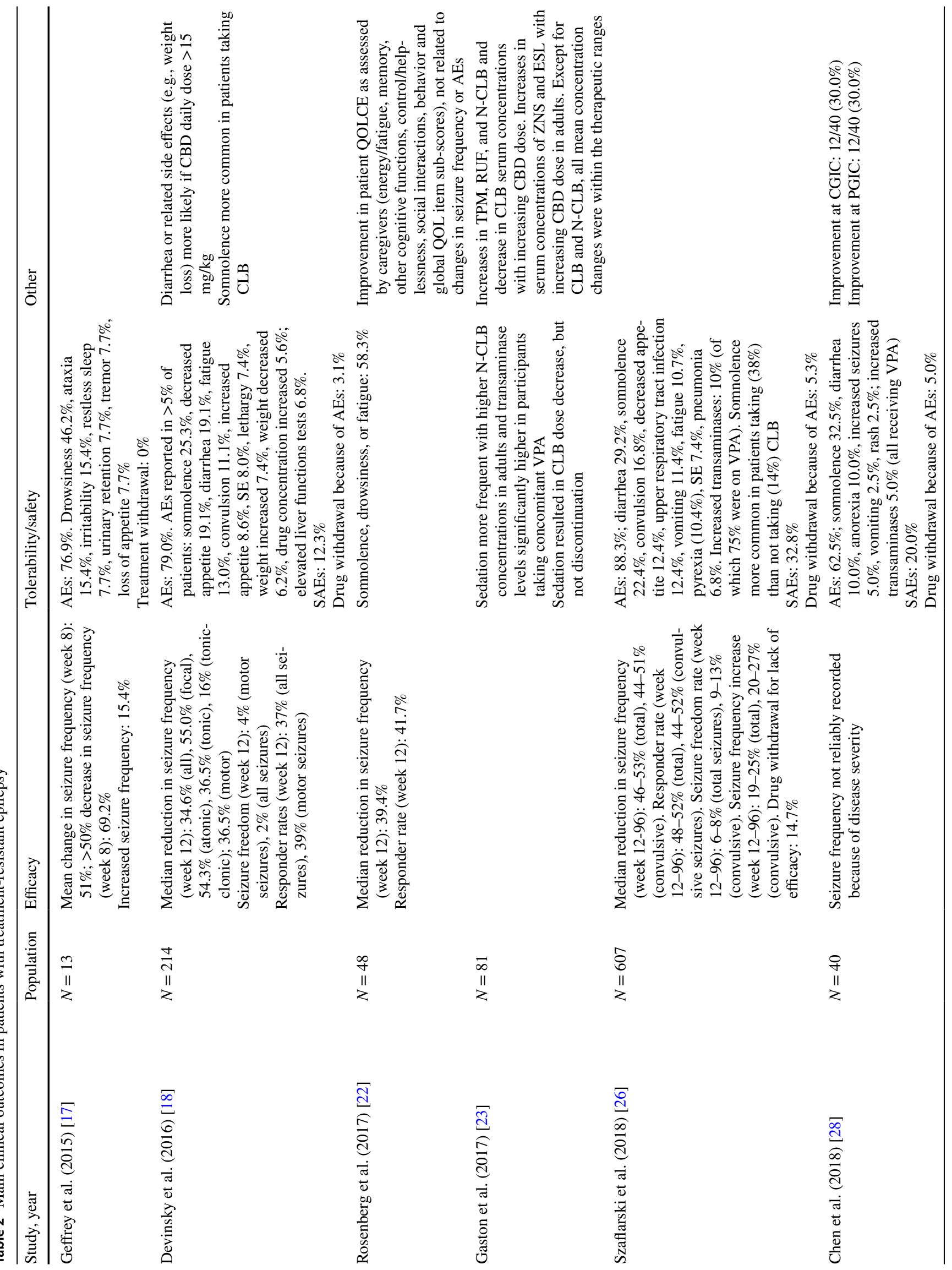




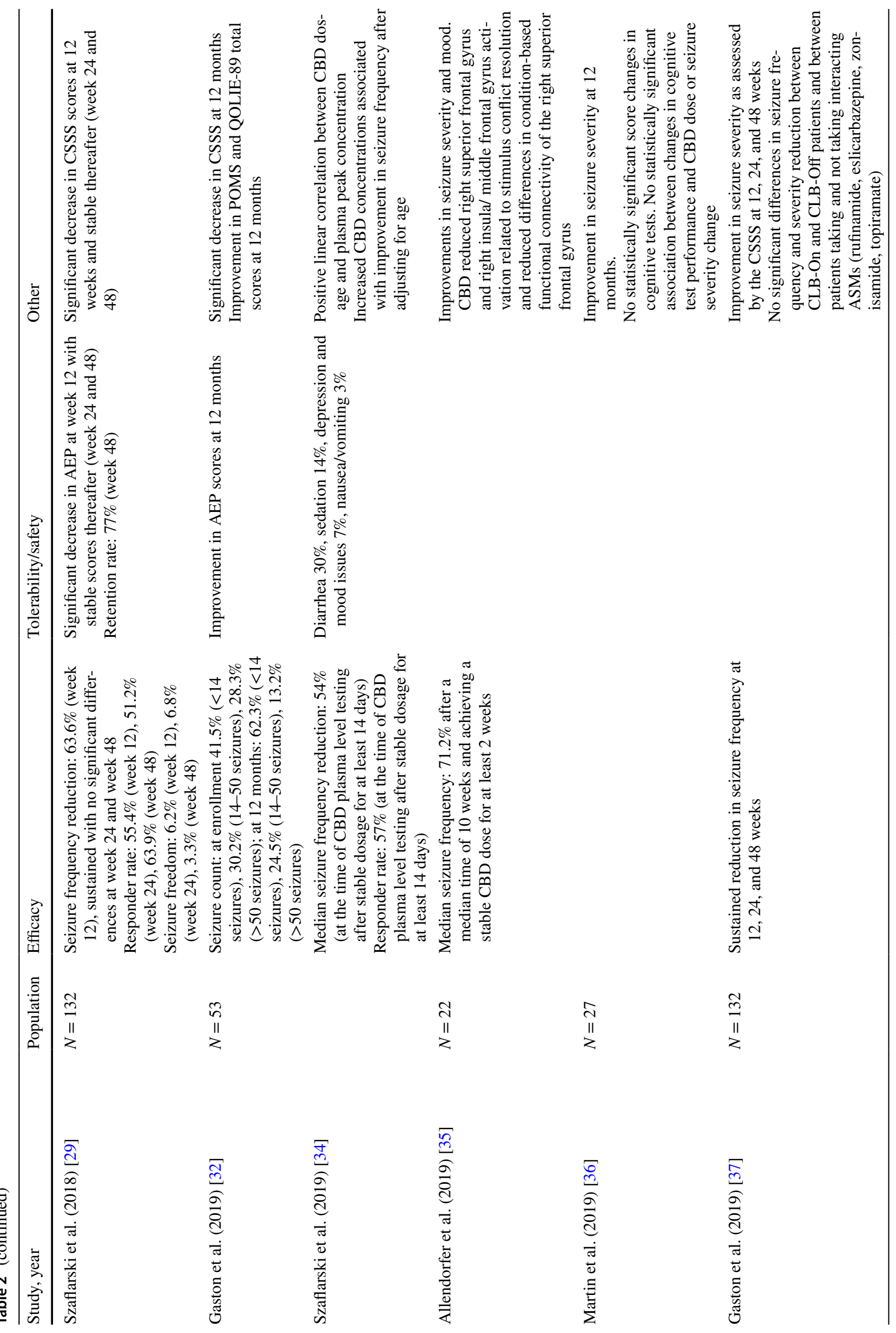




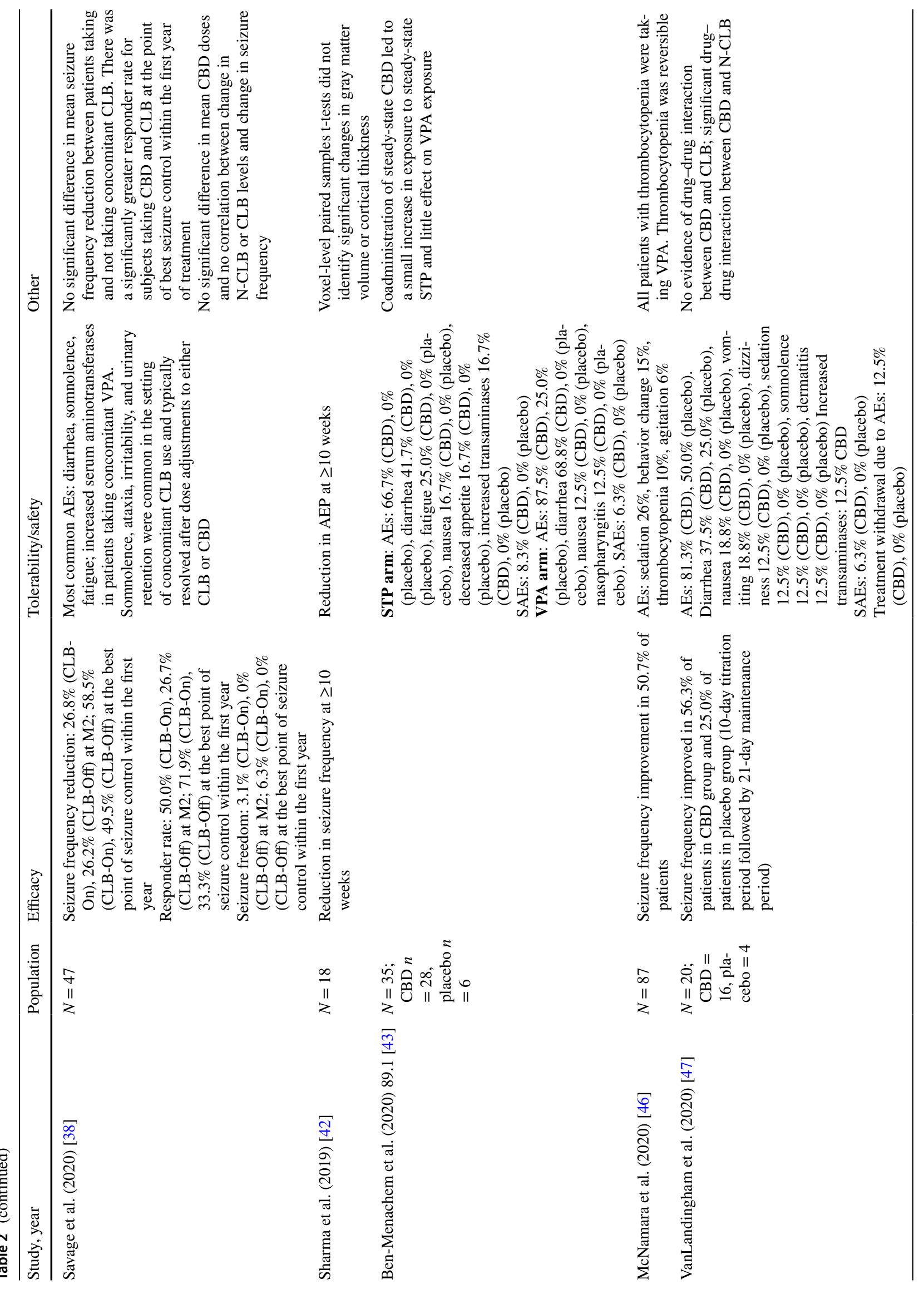




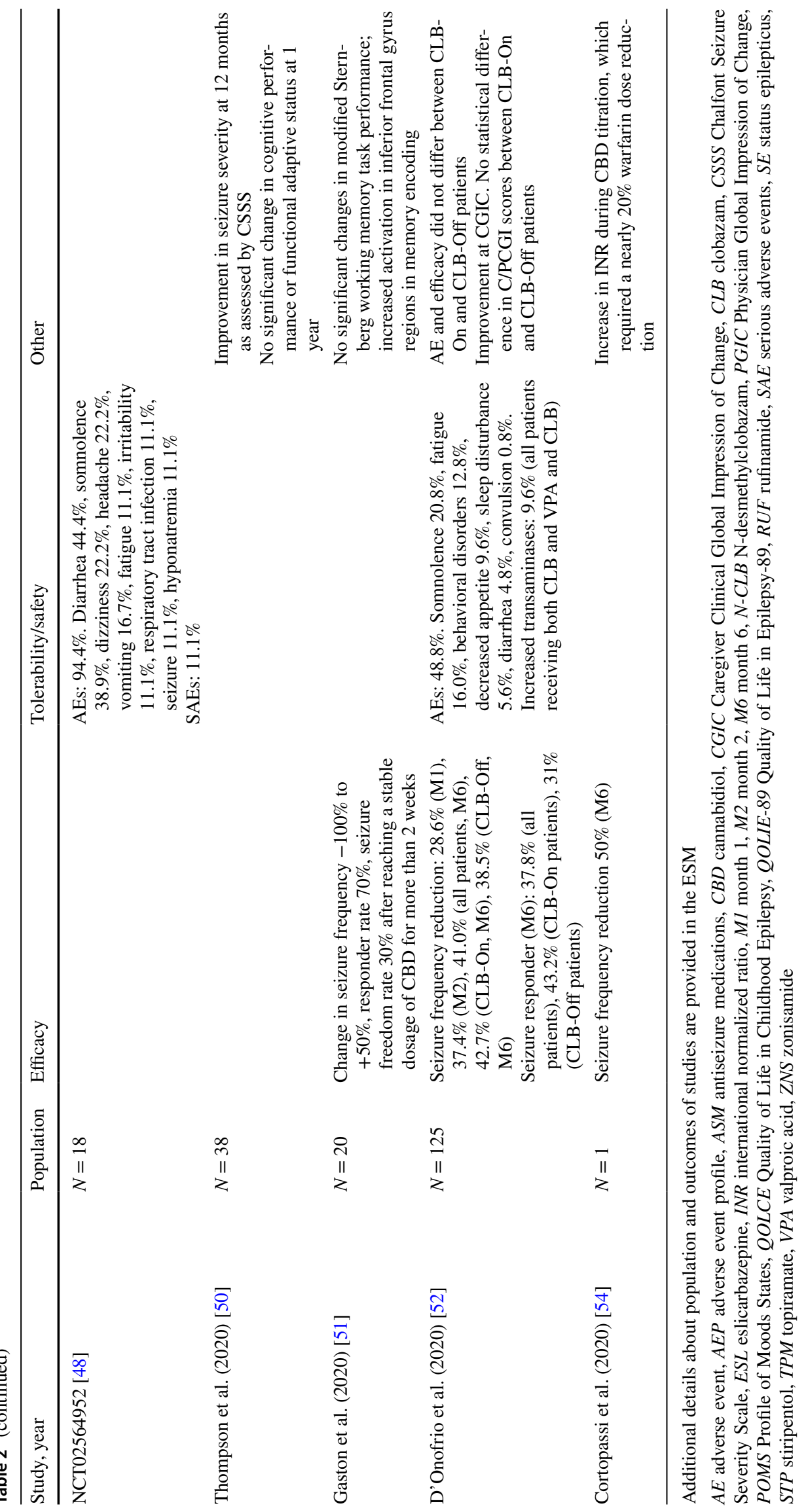


In the population of patients with medically refractory epilepsy, CBD given as an adjunct treatment at $5-50 \mathrm{mg} / \mathrm{kg} /$ day exhibited an appreciable reduction in seizure frequency in a subset of patients with Sturge-Weber syndrome, focal cortical dysplasia, lissencephaly, brain tumor-related epilepsy, and frontal and temporal lobe epilepsy of unknown etiology. Finally, in the setting of emergency and urgency, pharmaceutical-grade purified CBD proved beneficial in a series of children treated for highly refractory seizures attributed to febrile infection-related epilepsy syndrome and individual cases of refractory status epilepticus.

The overall tolerability profile of purified CBD was favorable across the different epileptic conditions and substantially overlapped that reported in patients with DS and LGS enrolled in randomized controlled trials [10,11]. The most common adverse events observed during treatment included somnolence, gastrointestinal symptoms, decreased appetite, and weight loss.

The incidence of somnolence was generally higher in patients concomitantly taking CLB. The increased serum level of $\mathrm{N}$-desmethyl-clobazam, the active metabolite of CLB, via inhibition of cytochrome P450 2C19 by CBD is likely to contribute to the risk [59]. Slow titration of CBD and dose adjustment of CLB can alleviate the side effect when the two drugs are concomitantly administered [52]. Further, as it is not easy to distinguish benzodiazepine toxicity from CBD-related adverse events on a clinical basis, therapeutic drug monitoring may be recommended before CBD administration and after any dose increase [60].

Gastrointestinal symptoms were generally mild to moderate in severity and usually evident within the first months of treatment. The sesame oil-based drug vehicle and the effects of CBD on the gut microbiome may contribute to diarrhea [61]. The lack of appetite and decreased weight are thought to be directly related to CBD as they occurred also independently from diarrhea [30]. Clinically significant weight loss emerged typically only after about 6 months of treatment.

The currently available data indicate no adverse effects involving cognitive or adaptive functioning related to treatment with purified CBD $[36,50]$. Improvement in cognition, behavior, mood, and multiple domains of health-related quality of life has been reported during CBD treatment in patients with TSC [20], children and young adults with severe childhood-onset epilepsy [22], and adult patients with refractory epilepsy [32]. These effects were independent of the reduction in seizure burden and may result from the changes induced by $\mathrm{CBD}$ in the connectivity related to executive functioning and emotional and attentional control processes, as observed in functional imaging studies [35, 49, 51]. Further, the lack of any impact on brain morphometry in terms of gray matter volume and cortical thickness changes despite positive action at molecular targets supports the safety of CBD in patients with treatment-resistant epilepsy, at least in the short and intermediate term [42].

Elevation of liver function tests occurred during CBD treatment. Most of the patients who experienced a rise in serum transaminases were concomitantly taking valproic acid (VPA). As CBD does not affect systemic concentrations of VPA or its metabolite, 4-ene-VPA [43], the interaction between CBD and VPA is most likely pharmacodynamic rather than pharmacokinetic. The interaction observed at the level of in vitro hepatic mitochondria could be the mechanism at the basis of the clinical findings [62, 63]. Interestingly, several patients who presented with elevations of liver function tests during the expanded access program were retitrated to baseline CBD dose after the withdrawal of VPA and did not experience any further laboratory abnormalities $[23,26]$. Given the risk of hepatocellular injury, slow uptitration and close monitoring of liver enzymes are recommended, mostly during the initial phases of treatment, in patients taking VPA, following changes in the CBD dose and the addition of or changes in medications with known effect on the liver, such as VPA and CLB [64].

A drug-drug interaction that can have clinical relevance is that between CBD and the mechanistic target of rapamycin inhibitors, which are used as treatment options for TSCassociated manifestations, including subependymal giant cell astrocytomas, renal angiomyolipomas, and focal seizures. Cannabidiol can result in increased serum concentrations of sirolimus (or rapamycin) and everolimus, likely via inhibition of cytochrome P450 3A4 [39]. Close monitoring of mechanistic target of rapamycin inhibitor serum concentrations, safety laboratory studies, and occurrence of adverse events is warranted, and a dose reduction may be required. The increase in mechanistic target of rapamycin inhibitor levels can be particularly significant in patients concomitantly treated with the ketogenic diet, as both treatments are associated with similar metabolic derangements [65].

While there was no treatment-related thrombocytopenia during the phase III trials, a single-center systematic chart review reported this adverse event in $10 \%$ of the pediatric (aged $<21$ years) patients diagnosed with LGS, DS, or other treatment-resistant epilepsies who were prescribed pharmaceutical-grade CBD [46]. Although all patients were concurrently treated with CBD and VPA, thrombocytopenia occurred independently of VPA doses and concentrations, and it was usually reversible with adjustments in the dosage of CBD or VPA; one case recovered spontaneously. If this finding is confirmed, monitoring for thrombocytopenia when adding CBD to a regimen that includes VPA may be also warranted.

The characteristics of the studies comprised in this review need to be considered in the interpretation of the findings. Major limitations included the open-label and uncontrolled 
design of the majority of the studies. The lack of blinding and comparator groups increases the potential for bias owing to the variable natural history of seizures and the high expectations for treatment [66]. The issue of the placebo response can be especially relevant in paediatric studies of CBD treatment because of the intense media and family interest in the compound [67]. The expectation of efficacy effect, however, is rarely sustained over time, and studies reporting long-term follow-up data can reduce or control it. Parent-reported measures of quality of life and cognitive and behavioral changes may be subjective views of the caregiver rather than patient experiences, and additional studies are needed to assess the quality of life of caregivers alone or patients themselves [67]. Further, the limited study sizes limited the generalizability of the results and the determination of statistical significance.

\section{Conclusions}

The approval of CBD represents a milestone in the history of medical use of cannabinoids to treat seizure disorders. Pharmaceutical-grade CBD oral solution is the first product made directly from the cannabis plant rather than created synthetically to be authorized by regulatory agencies, and the first in a new class of antiseizure medications. Experimental data demonstrated that CBD may have antiseizure properties in a broad range of epilepsy syndromes and etiologies [68, 69], and open-label studies suggested the effectiveness of purified CBD in the treatment of children and adults presenting with other epilepsy syndromes and seizure types than those addressed by regulatory trials, including CDKL5 deficiency disorder and Aicardi, Dup15q, and Doose syndromes, SYNGAP1 encephalopathy, and Sturge-Weber syndrome. Of note, results cannot be directly transferred to other cannabisderived products and non-purified forms of medical marijuana or its components.

Epileptic encephalopathies are associated with treatmentresistant seizures, high medication burden, neurodevelopmental delays, and disabling comorbidities [70]. To date, there are no drugs specifically approved for these conditions, and existing therapies can determine or exacerbate cognitive, behavioral, and motor disorders [27]. The currently available evidence about pharmaceutical-grade CBD provides preliminary support for additional research. It is, however, worth noticing that clinical studies in orphan diseases necessarily vary from trials in non-rare conditions and are less likely to use randomization, blinding, and active comparators [71]. Considering the high morbidity and mortality associated with uncontrolled epilepsy and the challenge to generate significant statistical power within the framework of traditional randomized, controlled trials, novel trial designs should be considered [72].
Supplementary Information The online version contains supplementary material available at https://doi.org/10.1007/s40263-021-00807-y.

\section{Declarations}

Funding No funding was received for the conduct of this study. Open access funding was provided by Università Politecnica delle Marche within the CRUI-CARE Agreement.

Conflict of Interest Simona Lattanzi has received speaker's or consultancy fees from Eisai, UCB Pharma, and GW Pharmaceuticals and has served on advisory boards for Angelini Pharma, Arvelle Therapeutics, BIAL, and GW Pharmaceuticals. Eugen Trinka has received speaker's honoraria from UCB Pharma, Biogen, Gerot-Lannach, Bial, Eisai, Takeda, Newbridge, Sunovion Pharmaceuticals Inc., LivaNova, and Novartis; consultancy funds from UCB Pharma, Biogen, Gerot-Lannach, Bial, Eisai, Takeda, Newbridge, GW Pharmaceuticals, Sunovion Pharmaceuticals Inc., and Novartis; and directorship funds from Neuroconsult GmbH. Eugen Trinka's institution received grants from Biogen, Red Bull, Merck, UCB Pharma, European Union, FWF Österreichischer Fond zur Wissenschaftsförderung, and Bundesministerium für Wissenschaft und Forschung. Pasquale Striano received fees and research grants from GW Pharmaceuticals, Zogenyx, Biomarin, and Kolfarma s.r.l. Chiara Rocchi, Sergio Salvemini, and Mauro Silvestrini have no conflicts of interest that are directly relevant to the content of this study. Francesco Brigo acted as a consultant for Eisai.

Ethics Approval Not applicable.

Consent to Participate Not applicable.

Consent for Publication Not applicable.

Availability of Data and Material Not applicable.

Author Contributions SL drafted the concept for the article, analyzed the data, and wrote the manuscript. SL, CR, and SS performed the literature search. ET, PS, MS, and FB critically revised the work. All authors contributed to the final manuscript.

Open Access This article is licensed under a Creative Commons Attribution-NonCommercial 4.0 International License, which permits any non-commercial use, sharing, adaptation, distribution and reproduction in any medium or format, as long as you give appropriate credit to the original author(s) and the source, provide a link to the Creative Commons licence, and indicate if changes were made. The images or other third party material in this article are included in the article's Creative Commons licence, unless indicated otherwise in a credit line to the material. If material is not included in the article's Creative Commons licence and your intended use is not permitted by statutory regulation or exceeds the permitted use, you will need to obtain permission directly from the copyright holder. To view a copy of this licence, visit http://creativecommons.org/licenses/by-nc/4.0/.

\section{References}

1. Hirtz D, Thurman DJ, Gwinn-Hardy K, Mohamed M, Chaudhuri AR, Zalutsky R. How common are the "common" neurologic disorders? Neurology. 2007;68:326-37.

2. Cagnetti C, Lattanzi S, Foschi N, Provinciali L, Silvestrini M. Seizure course during pregnancy in catamenial epilepsy. Neurology. 2014;83:339-44. 
3. Lattanzi S, Zaccara G, Giovannelli F, Grillo E, Nardone R, Silvestrini $\mathrm{M}$, et al. Antiepileptic monotherapy in newly diagnosed focal epilepsy: a network meta-analysis. Acta Neurol Scand. 2019; 139:33-41.

4. Lattanzi S, Cagnetti C, Foschi N, Provinciali L, Silvestrini M. Lacosamide monotherapy for partial onset seizures. Seizure. 2015;27:71-4.

5. Kwan P, Brodie MJ. Early identification of refractory epilepsy. N Engl J Med. 2000;342:314-9.

6. Chen Z, Brodie MJ, Liew D, Kwan P. Treatment outcomes in patients with newly diagnosed epilepsy treated with established and new antiepileptic drugs: a 30-year longitudinal cohort study. JAMA Neurol. 2018;75:279-86.

7. Laxer KD, Trinka E, Hirsch LJ, Cendes F, Langfitt J, Delanty N, et al. The consequences of refractory epilepsy and its treatment. Epilepsy Behav. 2014;37:59-70.

8. Devinsky O, Cilio MR, Cross H, Fernandez-Ruiz J, French J, Hill C, et al. Cannabidiol: pharmacology and potential therapeutic role in epilepsy and other neuropsychiatric disorders. Epilepsia. 2014;55:791-802.

9. Cilio MR, Thiele EA, Devinsky O. The case for assessing cannabidiol in epilepsy. Epilepsia. 2014;55:787-90.

10. Lattanzi S, Brigo F, Trinka E, Zaccara G, Striano P, Del Giovane $\mathrm{C}$, et al. Adjunctive cannabidiol in patients with Dravet syndrome: a systematic review and meta-analysis of efficacy and safety. CNS Drugs. 2020;34:229-41.

11. Lattanzi S, Brigo F, Cagnetti C, Trinka E, Silvestrini M. Efficacy and safety of adjunctive cannabidiol in patients with LennoxGastaut syndrome: a systematic review and meta-analysis. CNS Drugs. 2018;32:905-16.

12. Lattanzi S, Trinka E, Russo E, Striano P, Citraro R, Silvestrini $\mathrm{M}$, et al. Cannabidiol as adjunctive treatment of seizures associated with Lennox-Gastaut syndrome and Dravet syndrome. Drugs Today (Barc). 2019;55:177-96.

13. ClinicalTrials.gov. A randomized controlled trial of cannabidiol (GWP42003-P, CBD) for seizures in tuberous sclerosis complex (GWPCARE6). ClinicalTrials.gov identifier: NCT02544763. 2020. https://clinicaltrials.gov/ct2/show/NCT02544763. Accessed Oct 2020.

14. ClinicalTrials.gov. Trial of cannabidiol (CBD; GWP42003-P) for infantile spasms (GWPCARE7). ClinicalTrials.gov identifier: NCT02953548. 2020. https://clinicaltrials.gov/ct2/show/NCT02 953548. Accessed Oct 2020.

15. ClinicalTrials.gov. Phase 3 trial of cannabidiol (CBD; GWP42003-P) for infantile spasms: open-label extensionpPhase (GWPCARE7). ClinicalTrials.gov identifier: NCT02954887. 2020. https://clinicaltrials.gov/ct2/show/NCT02954887. Accessed Oct 2020

16. Saade D, Joshi C. Pure cannabidiol in the treatment of malignant migrating partial seizures in infancy: a case report. Pediatr Neurol. 2015;52:544-7.

17. Geffrey AL, Pollack SF, Bruno PL, Thiele EA. Drug-drug interaction between clobazam and cannabidiol in children with refractory epilepsy. Epilepsia. 2015;56:1246-51.

18. Devinsky O, Marsh E, Friedman D, Thiele E, Laux L, Sullivan J, et al. Cannabidiol in patients with treatment-resistant epilepsy: an open-label interventional trial. Lancet Neurol. 2016;15:270-8.

19. Gofshteyn JS, Wilfong A, Devinsky O, Bluvstein J, Charuta J, Ciliberto MA, et al. Cannabidiol as a potential treatment for febrile infection-related epilepsy syndrome (FIRES) in the acute and chronic phases. J Child Neurol. 2017;32:35-40.

20. Hess EJ, Moody KA, Geffrey AL, Pollack SF, Skirvin LA, Bruno PL, et al. Cannabidiol as a new treatment for drug-resistant epilepsy in tuberous sclerosis complex. Epilepsia. 2016;57:1617-24.
21. Kaplan EH, Offermann EA, Sievers JS, Comi AM. Cannabidiol treatment for refractory seizures in Sturge-Weber syndrome. Pediatr Neurol. 2017;71(18-23):e2.

22. Rosenberg EC, Louik J, Conway E, Devinsky O, Friedman D. Quality of life in childhood epilepsy in pediatric patients enrolled in a prospective, open-label clinical study with cannabidiol. Epilepsia. 2017;58:e96-100.

23. Gaston TE, Bebin EM, Cutter GR, Liu Y, Szaflarski JP. UAB CBD Program. Interactions between cannabidiol and commonly used antiepileptic drugs. Epilepsia. 2017;58:1586-92.

24. Warren PP, Bebin EM, Nabors LB, Szaflarski JP. The use of cannabidiol for seizure management in patients with brain tumorrelated epilepsy. Neurocase. 2017;23:287-91.

25. Grayson L, Vines B, Nichol K, Szaflarski JP. UAB CBD Program. An interaction between warfarin and cannabidiol, a case report. Epilepsy Behav Case Rep. 2017;9:10-1.

26. Szaflarski JP, Bebin EM, Comi AM, Patel AD, Joshi C, Checketts D, et al. CBD EAP Study Group. Long-term safety and treatment effects of cannabidiol in children and adults with treatmentresistant epilepsies: expanded access program results. Epilepsia. 2018;59:1540-8.

27. Devinsky O, Verducci C, Thiele EA, Laux LC, Patel DA, Filloux $\mathrm{F}$, et al. Open-label use of highly purified CBD (Epidiolex $\left.{ }^{\circledR}\right)$ in patients with CDKL5 deficiency disorder and Aicardi, Dup15q, and Doose syndromes. Epilepsy Behav. 2018;86:131-7.

28. Chen KA, Farrar M, Cardamone M, Gill D, Smith R, Cowell CT, et al. Cannabidiol for treating drug-resistant epilepsy in children: the New South Wales experience. Med J Aust. 2018;209:217-21.

29. Szaflarski JP, Bebin EM, Cutter G, De Wolfe J, Dure LS, Gaston TE, et al. UAB CBD Program. Cannabidiol improves frequency and severity of seizures and reduces adverse events in an openlabel add-on prospective study. Epilepsy Behav. 2018;87:131-6.

30. Sands TT, Rahdari S, Oldham MS, Nunes EC, Tilton N, Cilio MR. Long-term safety, tolerability, and efficacy of cannabidiol in children with refractory epilepsy: results from an expanded access program in the US. CNS Drugs. 2019;33:47-60.

31. Rajaraman RR, Sankar R, Hussain SA. Successful use of pure cannabidiol for the treatment of super-refractory status epilepticus. Epilepsy Behav Case Rep. 2018;10:141-4.

32. Gaston TE, Szaflarski M, Hansen B, Bebin EM, Szaflarski JP. UAB CBD Program. Quality of life in adults enrolled in an openlabel study of cannabidiol (CBD) for treatment-resistant epilepsy. Epilepsy Behav. 2019;95:10-7.

33. Leino AD, Emoto C, Fukuda T, Privitera M, Vinks AA, Alloway RR. Evidence of a clinically significant drug-drug interaction between cannabidiol and tacrolimus. Am J Transpl. 2019;19:2944-8.

34. Szaflarski JP, Hernando K, Bebin EM, Gaston TE, Grayson LE, Ampah SB, et al. Higher cannabidiol plasma levels are associated with better seizure response following treatment with a pharmaceutical grade cannabidiol. Epilepsy Behav. 2019;95:131-6.

35. Allendorfer JB, Nenert R, Bebin EM, Gaston TE, Grayson LE, Hernando KA, et al. fMRI study of cannabidiol-induced changes in attention control in treatment-resistant epilepsy. Epilepsy Behav. 2019;96:114-21.

36. Martin RC, Gaston TE, Thompson M, Ampah SB, Cutter G, Bebin $\mathrm{EM}$, et al. Cognitive functioning following long-term cannabidiol use in adults with treatment-resistant epilepsy. Epilepsy Behav. 2019;97:105-10.

37. Gaston TE, Bebin EM, Cutter GR, Ampah SB, Liu Y, Grayson LP, et al. UAB CBD Program. Drug-drug interactions with cannabidiol (CBD) appear to have no effect on treatment response in an open-label Expanded Access Program. Epilepsy Behav. 2019;98(Pt A):201-6.

38. Savage TE, Sourbron J, Bruno PL, Skirvin LA, Wolper ES, Anagnos CJ, et al. Efficacy of cannabidiol in subjects with refractory 
epilepsy relative to concomitant use of clobazam. Epilepsy Res. 2020;160:106263.

39. Ebrahimi-Fakhari D, Agricola KD, Tudor C, Krueger D, Franz DN. Cannabidiol elevates mechanistic target of rapamycin inhibitor levels in patients with tuberous sclerosis complex. Pediatr Neurol. 2020;105:59-61.

40. Poisson K, Wong M, Lee C, Cilio MR. Response to cannabidiol in epilepsy of infancy with migrating focal seizures associated with KCNT1 mutations: an open-label, prospective, interventional study. Eur J Paediatr Neurol. 2020;25:77-81.

41. Herlopian A, Hess EJ, Barnett J, Geffrey AL, Pollack SF, Skirvin L, et al. Cannabidiol in treatment of refractory epileptic spasms: an open-label study. Epilepsy Behav. 2020;106:106988.

42. Sharma AA, Nenert R, Allendorfer JB, Gaston TE, Grayson LP, Hernando K, et al. A preliminary study of the effects of cannabidiol (CBD) on brain structure in patients with epilepsy. Epilepsy Behav Rep. 2019;12:100341.

43. Ben-Menachem E, Gunning B, Cabrera CMA, Van Landingham $\mathrm{K}$, Crockett J, Critchley D, et al. A phase II randomized trial to explore the potential for pharmacokinetic drug-drug interactions with stiripentol or valproate when combined with cannabidiol in patients with epilepsy. CNS Drugs. 2020;34:661-72.

44. Barnett JR, Grinspoon RA, Harisinghani M, Caruso PA, Thiele EA. The efficacy of cannabidiol on renal angiomyolipoma and subependymal giant cell tumor volume in tuberous sclerosis complex. J Clin Neurosci. 2020;77:85-8.

45. Gupta S, Schwab M, Valdez-Gonzalez K, Segal E. Rare homozygous nonsense variant in AIMP1 causing early onset epileptic encephalopathy with burst Ssuppression (EOEE-BS). Eur J Med Genet. 2020;63:103970.

46. McNamara NA, Dang LT, Sturza J, Ziobro JM, Romanowski EMF, Smith GC, et al. Thrombocytopenia in pediatric patients on concurrent cannabidiol and valproic acid. Epilepsia. 2020;61:e85-9.

47. Van Landingham KE, Crockett J, Taylor L, Morrison G. A phase 2, double-blind, placebo-controlled trial to investigate potential drug-drug interactions between cannabidiol and clobazam. J Clin Pharmacol. 2020;60:1304-13.

48. ClinicalTrials.gov. An open-label extension study to investigate possible drug-drug interactions between clobazam and cannabidiol. ClinicalTrials.gov identifier: NCT02564952. 2020. https:// clinicaltrials.gov/ct2/show/NCT02564952. Accessed Oct 2020.

49. Nenert R, Allendorfer JB, Bebin EM, Gaston TE, Grayson LE, Houston JT, et al. Cannabidiol normalizes resting-state functional connectivity in treatment-resistant epilepsy. Epilepsy Behav. 2020;112:107297.

50. Thompson MD, Martin RC, Grayson LP, Ampah SB, Cutter G, Szaflarski JP, et al. Cognitive function and adaptive skills after a one-year trial of cannabidiol (CBD) in a pediatric sample with treatment-resistant epilepsy. Epilepsy Behav. 2020;111:107299.

51. Gaston TE, Allendorfer JB, Nair S, Bebin EM, Grayson LP, Martin RC, et al. UAB CBD Program. Effects of highly purified cannabidiol (CBD) on fMRI of working memory in treatmentresistant epilepsy. Epilepsy Behav. 2020;112:107358.

52. D’Onofrio G, Kuchenbuch M, Hachon-Le Camus C, Desnous B, Staath V, Napuri S, et al. Slow titration of cannabidiol add-on in drug-resistant epilepsies can improve safety with maintained efficacy in an open-label study. Front Neurol. 2020;11:829.

53. Kuchenbuch M, D'Onofrio G, Chemaly N, Barcia G, Teng T, Nabbout R. Add-on cannabidiol significantly decreases seizures in 3 patients with SYNGAP1 developmental and epileptic encephalopathy. Epilepsia Open. 2020;5:496-500.

54. Cortopassi J. Warfarin dose adjustment required after cannabidiol initiation and titration. Am J Health Syst Pharm. 2020;77:1846-51.
55. Bellon M, Walker C, Peterson C. Seizure-related injuries and hospitalizations: self-report data from the 2010 Australian Epilepsy Longitudinal Survey. Epilepsy Behav. 2013;26:7-10.

56. Cortright DN, Szallasi A. Biochemical pharmacology of the vanilloid receptor TRPV1. Eur J Biochem. 2004;271:1814-9.

57. Sun F-J, Guo W, Zheng D-H, Zhang C-Q, Li S, Liu S-Y, et al. Increased expression of TRPV1 in the cortex and hippocampus from patients with mesial temporal lobe epilepsy. J Mol Neurosci. 2013;49:182-93.

58. Lattanzi S, Trinka E, Striano P, Zaccara G, Del Giovane C, Nardone R, et al. Cannabidiol efficacy and clobazam status: a systematic review and meta-analysis. Epilepsia. 2020;61:1090-8.

59. Lattanzi S, Zaccara G, Russo E, La Neve A, Lodi MAM, Striano P. Practical use of pharmaceutically purified oral cannabidiol in Dravet syndrome and Lennox-Gastaut syndrome. Expert Rev Neurother. 2020. https://doi.org/10.1080/14737175.2021.18343 83 (Epub ahead of print).

60. Skinner CM, Nookaew I, Ewing LE, Wongsurawat T, Jenjaroenpun P, Quick CM, et al. Potential probiotic or trigger of gut inflammation: the Janus-faced nature of cannabidiol-rich cannabis extract. J Diet Suppl. 2020;17:543-60.

61. FDA briefing document. Peripheral and central nervous system drugs. Advisory Committee Meeting. April 19, 2018. NDA210365. Cannabidiol. 2018. https://www.fda.gov/media/ 112565/download. Accessed Dec 2020.

62. Lattanzi S, Brigo F, Trinka E, Zaccara G, Cagnetti C, Del Giovane $\mathrm{C}$, et al. Efficacy and safety of cannabidiol in epilepsy: a systematic review and meta-analysis. Drugs. 2018;78:1791-804.

63. Epidiolex (cannabidiol) oral solution: highlights of prescribing information. 2020. https://www.accessdata.fda.gov/drugsatfda docs/label/2018/210365lbl.pdf. Accessed Dec 2020.

64. Busaidy NL, Farooki A, Dowlati A, Perentesis JP, Dancey JE, Doyle LA, Brell JM, Siu LL. Management of metabolic effects associated with anticancer agents targeting the PI3K-Akt-mTOR pathway. J Clin Oncol. 2012;30:2919-28.

65. Zaccara G, Giovannelli F, Schmidt D. Placebo and nocebo responses in drug trials of epilepsy. Epilepsy Behav. 2015;43:128-34.

66. Press CA, Knupp KG, Chapman KE. Parental reporting of response to oral cannabis extracts for treatment of refractory epilepsy. Epilepsy Behav. 2015;45:49-52.

67. Jones NA, Glyn SE, Akiyama S, Hill TD, Hill AD, Weston SE, et al. Cannabidiol exerts anti-convulsant effects in animal models of temporal lobe and partial seizures. Seizure. 2012;21:344-52.

68. Consroe P, Wolkin A. Cannabidiol-antiepileptic drug comparisons and interactions in experimentally induced seizures in rats. J Pharmacol Exp Ther. 1977;201:26-32.

69. Scheffer IE, Berkovic S, Capovilla G, Connolly M, French J, Guilhoto L, et al. ILAE classification of the epilepsies: position paper of the ILAE Commission for Classification and Terminology. Epilepsia. 2017;4:512-21.

70. Logviss K, Krievins D, Purvina S. Characteristics of clinical trials in rare vs. common diseases: a register-based Latvian study. PLoS One. 2018;13:e0194494.

71. Margolis A, Giuliano C. Making the switch: from case studies to N-of-1 trials. Epilepsy Behav Rep. 2019;12:100336.

72. Ong KS, Carlin JB, Fahey M, Freeman JL, Scheffer IE, Gillam L, et al. Protocol for a single patient therapy plan: a randomised, double-blind, placebo-controlled N-of-1 trial to assess the efficacy of cannabidiol in patients with intractable epilepsy. J Paediatr Child Health. 2020. https://doi.org/10.1111/jpc.15078 (Epub ahead of print). 


\section{Authors and Affiliations}

\section{Simona Lattanzi ${ }^{1}$ (D) Eugen Trinka ${ }^{2,3,4} \cdot$ Pasquale Striano $^{5} \cdot$ Chiara Rocchi $^{1} \cdot$ Sergio Salvemini ${ }^{1} \cdot$ Mauro Silvestrini ${ }^{1}$. Francesco Brigo ${ }^{6,7}$}

Simona Lattanzi

alfierelattanzisimona@gmail.com

1 Department of Experimental and Clinical Medicine, Neurological Clinic, Marche Polytechnic University, Via Conca 71, 60020 Ancona, Italy

2 Department of Neurology, Christian Doppler Klinik, Paracelsus Medical University, Salzburg, Austria

3 Center for Cognitive Neuroscience, Salzburg, Austria

4 Public Health, Health Services Research and HTA, University for Health Sciences, Medical Informatics and Technology, Hall in Tirol, Austria
5 Pediatric Neurology and Muscular Diseases Unit, Department of Neurosciences, Rehabilitation, Ophthalmology, Genetics, Maternal and Child Health, "G. Gaslini”" Institute, University of Genoa, Genoa, Italy

6 Department of Neuroscience, Biomedicine and Movement Science, University of Verona, Verona, Italy

7 Division of Neurology, "Franz Tappeiner" Hospital, Merano, BZ, Italy 